\title{
BMJ Open Patient feedback for safety improvement in primary care: results from a feasibility study
}

\author{
Andrea L Hernan (D) , ${ }^{1}$ Sally J Giles, ${ }^{2}$ Hannah Beks (D) , ${ }^{1}$ Kevin McNamara, ${ }^{1}$ \\ Kate Kloot, ${ }^{3}$ Marley J Binder, ${ }^{1}$ Vincent Versace ${ }^{1}$
}

To cite: Hernan AL, Giles SJ, Beks $\mathrm{H}$, et al. Patient feedback for safety improvement in primary care: results from a feasibility study. BMJ Open 2020;10:e037887. doi:10.1136/ bmjopen-2020-037887

- Prepublication history and additional material for this paper are available online. To view these files, please visit the journal online (http://dx.doi. org/10.1136/bmjopen-2020037887).

Received 20 February 2020 Revised 05 May 2020 Accepted 29 May 2020

\section{Check for updates}

C Author(s) (or their employer(s)) 2020. Re-use permitted under CC BY-NC. No commercial re-use. See rights and permissions. Published by BMJ.

${ }^{1}$ Deakin Rural Health, Deakin University, Geelong, Victoria, Australia

${ }^{2}$ NIHR Greater Manchester Primary Care Patient Safety Translational Research Centre, University of Manchester, Manchester, UK

${ }^{3}$ Centre for Rural Emergency Medicine, Deakin University, Warrnambool, Victoria, Australia

Correspondence to

Dr Andrea L Hernan;

andrea.hernan@deakin.edu.au

\section{ABSTRACT}

Objectives Patient involvement in safety improvement is a developing area of research. The aim of this study was to investigate the feasibility of a patient feedback on safety intervention in primary care. Specifically, the intervention acceptability, fidelity, implementation enablers and barriers, scalability, and process of systematically collecting safety data were examined.

Design, setting and participants Mixed-methods feasibility trial with six purposively selected Australian primary care practices.

Intervention The intervention comprised an iterative process with a cycle of measurement, learning, feedback, action planning and implementation period of 6 months.

Primary and secondary outcomes Qualitative and quantitative data relating to feasibility measures (acceptability, fidelity, enablers, barriers, scalability and process of collecting safety data) were collected and analysed.

Results A total of $n=1750$ patients provided feedback on safety. There was a statistically significant increase in mean patient safety scores indicating improved safety (4.30-4.37, $p=0.002)$. Staff deemed the intervention acceptable, with minor recommendations for improvement. Intervention fidelity was high and implementation enablers were attributed to the intervention structure and framework, use of intuitive problem-solving approaches, and multidisciplinary team involvement. Practicebased safety interventions resulted in sustainable and measurable changes to systems for safety, such as increased access to care and improved patient information accuracy.

Conclusions The findings indicate that this innovative patient feedback on safety intervention is feasible for scale-up to a larger effectiveness trial and further spread into policy and practice. This intervention complements existing safety improvement strategies and activities, and integrates into current patient feedback service requirements for Australian primary care. Further research is needed to examine the intervention effects on safety incident reduction.

\section{INTRODUCTION}

Involving patients in error prevention and harm reduction activities has gained traction over the past decade. ${ }^{1-7}$ Patient engagement has been found to prevent or reduce adverse

\section{Strengths and limitations of this study}

A feasibility study was conducted prior to the development and implementation of a large-scale effectiveness trial and wider spread and uptake into policy and practice.

- Several feasibility domains were assessed including intervention acceptability, fidelity, implementation enablers and barriers, scalability, and process of systematically collecting safety data in a primary care.

- A mixed-methods approach addressed each feasibility domain and included both qualitative and quantitative data collection and analysis.

- A limitation is that the data collected will be mostly descriptive, and therefore, the generalisability of the findings may be limited to only one geographical area.

events, and increase awareness of potential safety risks. ${ }^{8}$ Much of this research has centred on hospital settings with the majority of interventions utilising patient feedback mechanisms for safety improvement. ${ }^{38-12}$ The evidence base regarding patient feedback on safety in primary care is considerably lacking by comparison.

In addition to reporting formal safety incidents, ${ }^{13-16}$ patient feedback about processes, systems and structures that lead to safety incidents is an essential piece of the safety intelligence 'jigsaw'. ${ }^{17}$ Patients have demonstrated understanding and knowledge about the various conditions in the latent environment that influence safety, such as access to care; communication systems; information and care planning; and transitions between care settings. ${ }^{17-20}$ Capturing patient feedback about these contributory factors to safety incidents and using it for safety improvement work in primary care is a developing and novel field of research. ${ }^{21}$

Only one validated, real-time and theoryderived patient feedback tool for assessment of factors contributing to safety in primary care 
is currently available - the primary care patient measure of safety (PC PMOS).$^{20} 22$ The PC PMOS aims to enhance or complement current data collection methods for patient safety in primary care. ${ }^{20}{ }^{22}$ This self-administered tool is an acceptable, efficient and appropriate mechanism for engaging patients in safety improvement. ${ }^{11} 131723$ The PC PMOS also facilitates primary care professionals and organisations learning, and drives implementation of real-time service improvements. ${ }^{2021}$

The implementation and impact of interventions which use the PC PMOS tool for data-driven improvement and ongoing safety monitoring in primary care remains unexplored. Primary care, like most healthcare settings, is a complex system with multiple and multilevel factors likely to affect implementation of a patient feedback for safety improvement intervention. ${ }^{24}$ While common barriers and enablers to implementation of quality and safety improvement interventions have been published, ${ }^{25-27}$ the specific processes and outcomes of using the PC PMOS in a primary care safety improvement intervention is unknown. Advocates for complexity science and implementation science in healthcareimprovement-research recommend feasibility studies be conducted prior to the introduction of large-scale effectiveness trials or wider spread into policy and practice. ${ }^{24}$ 25 28-30 Therefore, the aim of this study was to understand the acceptability, fidelity, implementation enablers and barriers, scalability, and process of systematically collecting safety data in a primary care patient feedback on safety intervention.

\section{METHODS}

A detailed description of the study design and sampling frame, intervention, and primary and secondary outcome measures has been published in the study protocol. ${ }^{21} \mathrm{~A}$ brief overview is provided below.

\section{Study design and sampling frame}

This was a mixed-methods feasibility trial with six purposively sampled primary care practices from the southwest region of Victoria, Australia (online supplementary appendix 1).

\section{Intervention}

\section{Intervention tool: PC PMOS}

The PC PMOS tool is an anonymous 28 item survey covering nine latent conditions in the primary care environment influencing safety incidents including: access to care, communication, the external policy environment, information flow, organisation and care planning, patientrelated factors, the physical environment, referral systems and task performance (available on request). ${ }^{20} 22$ The PC PMOS consists of a five-point Likert scale with higher scores indicating safer primary care. The PC PMOS also captures patient-reported safety incident data.

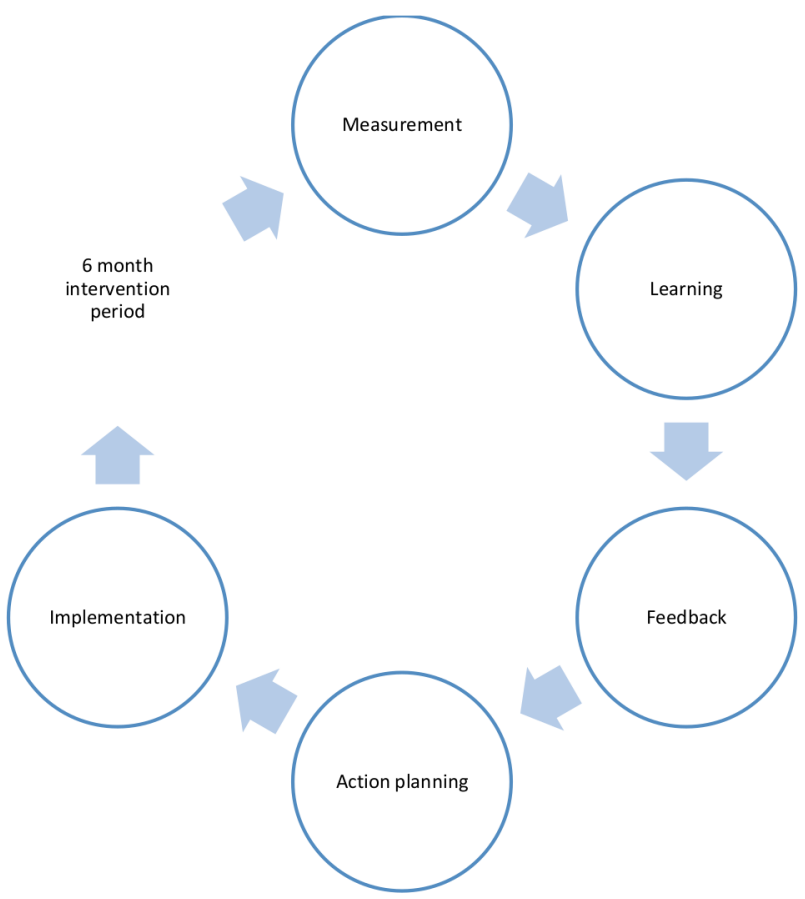

Figure 1 Intervention phases.

\section{Intervention phases}

The intervention comprised an iterative process with a cycle of measurement, learning, feedback, action planning and implementation period of 6 months (figure 1 ).

Patient feedback about the safety of their care was measured using the PC PMOS tool at baseline (time 1-T1). Primary care teams then used patient feedback from the PC PMOS to develop and implement specific safety interventions over a 6-month period. Patient feedback about the safety of their care was measured again (PC PMOS) at the end of the intervention period (time 2-T2).

Primary care practices were asked to form safety improvement teams (SIT). These teams comprised a minimum of three members and included any combination of practice manager, practice nurse, receptionist or administration staff or general practitioner.

SIT members participated in two learning and development workshops on teamwork, communication, implementation planning, the model for improvement's (MfI) Plan-Do-Study-Act (PDSA) methodology ${ }^{31}$ and trial information.

PC PMOS data from each practice were collated and presented to the SIT at an action planning meeting. SIT members considered which area(s) of safety improvement to target, and developed goals, measures, ideas and PDSA cycles. SIT members were responsible for implementing and monitoring their specific safety intervention/s through application of multiple PDSA cycles over the 6-month period.

\section{Data collection}

Primary outcome

Feasibility measures included acceptability, intervention fidelity, implementation enablers and barriers, and 
scalability. These data were collected using three qualitative methods:

- Recordings and overt observations of SIT members at workshops and action planning meetings,

- Semistructured interviews with SIT members at trial conclusion,

- Reflexive researcher journaling.

Audio data were transcribed verbatim. Overt participant observation data were recorded using detailed field note diaries and regular researcher discussion and reflection. Approximately 31 hours of audio was recorded with participants at workshops $(2 \times 3$ hours $)$, action planning meetings $(6 \times 1.5$ hours $)$ and semistructured interviews (16 hours-13 discrete individual or group interviews).

\section{Secondary outcomes}

Patient feedback on contributing factors to safety

Every adult ( $\geq 18$ years) presenting for their appointment was invited by the practice receptionist to complete the PC PMOS over a 3-week period. Patients returned their surveys via a secure survey return box in the practice waiting room. Surveys were anonymous and completion was voluntary.

\section{Patient-reported safety incidents and concerns}

The PC PMOS contains questions for patients to report any patient safety incident. Questions were adapted from the 'Patient Incident Reporting Tool' used in the Patient Reporting and Action for a Safe Environment intervention. ${ }^{32}$ The PC PMOS has an 'other comments' free-text question which also provides patients the opportunity to report safety incidents or concerns.

\section{Staff safety culture}

The validated Agency for Healthcare Research and Quality Medical Office Survey (MOS) on patient safety ${ }^{33}$ was used to obtain data about staff safety culture perceptions at baseline (T1) (prior to patient data collection) and after the intervention (T2). All staff were invited to complete the survey and return it to the researcher via a provided prepaid envelope. Surveys were anonymous and completion was voluntary.

\section{Safety incident reports}

Practice managers provided a deidentified copy of their practice's clinical risk management/safety incident register from the previous 12 months at T1 and T2. Due to lack of detailed data provided on the register, specific analysis or categorisation of the safety incidents was unable to be performed. However, the type of incident and any patient demographic data (age, gender) were cross checked with the patient-reported safety incidents on the PC PMOS to assess for similarities or differences.

\section{Data analysis}

Primary outcome

Triangulation and thematic analysis techniques were employed to analyse the qualitative and content data. Both inductive and deductive approaches were used to undertake the analysis. ${ }^{34}$ Deductive approaches used the literature about healthcare culture and safety improvement, patient feedback and response theory, health service implementation science, and engagement and adaption theory. ${ }^{25-27} 3536$ Inductive coding was also performed on qualitative and content data by three researchers (ALH, SJG and HB). The initial coding framework centred on the feasibility measures of intervention enablers, barriers, acceptability, fidelity and scalability. This framework was expanded through constant comparison with the data to create the final coding framework. Discrepancies between researchers were resolved through discussion. NVivo (QSR International) was used to support the analysis.

\section{Intervention fidelity score}

Intervention fidelity refers to the implementation of safety improvement interventions being delivered as intended. ${ }^{37}$ The number of safety interventions implemented at each practice was assessed by the research team using a three choice response option-yes, no or partially.

\section{Secondary outcomes}

Quantitative data were analysed using SPSS Statistics (IBM V.24). Continuous variables were compared preintervention and postintervention using t-tests, while comparisons for non-parametric data used the Mann-Whitney $\mathrm{U}$ test. Categorical variables were compared using $\mathrm{X}^{2}$-tests. Results were considered statistically significant where $\mathrm{p} \leq 0.05$.

The MOS percent positive scores for each 10 patient safety culture composites, the average score across the 10 composites, and the overall patient safety rating were calculated at $\mathrm{T} 1$ and $\mathrm{T} 2$ for each practice, and overall using t-tests.

\section{Patient and public involvement}

Patients directly participated in the priority setting of safety interventions at a local level. Specifically, patients concerns or experiences with systems for safety in the primary care environment (eg, access to care, communication, information and referral processes, organisation and care planning) were acted on by primary care teams through development and implementation of interventions which prevent safety incidents from occurring.

\section{Informed consent}

Patient consent was implied by completion and return of the PC PMOS questionnaire. This was stated on the plain language statement accompanying the PC PMOS questionnaire. All staff who participated in a semistructured interview with the researchers provided written consent to participate. Each practice manager provided written practice consent for the research to be undertaken at their practice.

\section{RESULTS}

\section{Primary outcome}

Representative participant quotes corresponding to feasibility measures are presented in table 1 . 
Table 1 Key participant quotes corresponding to feasibility measures

\begin{tabular}{|c|c|c|c|}
\hline $\begin{array}{l}\text { Feasibility } \\
\text { measure }\end{array}$ & Theme & Subtheme & Participant quote \\
\hline \multirow[t]{9}{*}{ Acceptability } & \multirow[t]{6}{*}{$\begin{array}{l}\text { Attitude towards } \\
\text { patient feedback } \\
\text { on safety }\end{array}$} & $\begin{array}{l}\text { Value patient feedback } \\
\text { on safety }\end{array}$ & $\begin{array}{l}\text { 'It's always, the valuable ones are always the awful ones, aren't they? You know... it's really precious. Ain't } \\
\text { often people are honest like that...' (GP, Practice D, APM) } \\
\text { 'It's better to be informed about it so that you can make that change....it makes it more positive for } \\
\text { everybody then'...(Administration Staff, Practice E, APM) }\end{array}$ \\
\hline & & $\begin{array}{l}\text { Patient feedback on } \\
\text { safety aligned with staff } \\
\text { awareness of issues }\end{array}$ & $\begin{array}{l}\text { 'the bits that were flagged that were in there [feedback report] were probably what we expected ...' (PN, } \\
\text { Practice D, APM). }\end{array}$ \\
\hline & & $\begin{array}{l}\text { Believability of the } \\
\text { feedback }\end{array}$ & 'And I accept the [safety incident] one, because, perception is truth.' (GP, Practice B, APM). \\
\hline & & $\begin{array}{l}\text { Concern and empathy } \\
\text { towards patient feedback }\end{array}$ & $\begin{array}{l}\text { '... there's one [safety incident] I was actually concerned, there's a patient who obviously feels that we } \\
\text { haven't done our best by them.' (PM, Practice A, APM). } \\
\text { 'So someone had a blocked airway. That sounds really terrible, doesn't it? It's (an) emergency.' (PN, Practice } \\
\text { A, APM). }\end{array}$ \\
\hline & & $\begin{array}{l}\text { Surprised or unsure } \\
\text { how to respond when } \\
\text { feedback differed to staff } \\
\text { perceptions }\end{array}$ & $\begin{array}{l}\text { 'I thought we have got some more negative feedback from people, which surprised me.' (PN1, Practice C, } \\
\text { APM). } \\
\text { 'But I'm not quite sure about that [safety incident] one... I found that one very odd, because... probably } \\
\text { some of the best staff we have are down that end of the building, without being horrible to others, but the } \\
\text { doctors even say that. I just find that really odd.' (PM, Practice B, APM). }\end{array}$ \\
\hline & & $\begin{array}{l}\text { Dismissive towards patient } \\
\text { feedback }\end{array}$ & $\begin{array}{l}\text { 'I think sometimes it's that lack of understanding, that they [GP] can't come and fix the world in fifteen } \\
\text { minutes' (PN1, Practice C, APM). } \\
\text { 'when you get that: 'I can't see the doctor that I want to see' [patient comment]. Well we've been working on } \\
\text { this for five years trying to improve things!' (PM, Practice D, Final Interview) }\end{array}$ \\
\hline & \multirow[t]{3}{*}{$\begin{array}{l}\text { Using patient } \\
\text { feedback to make } \\
\text { changes }\end{array}$} & $\begin{array}{l}\text { Cautious about using } \\
\text { patient feedback for safety } \\
\text { improvement }\end{array}$ & $\begin{array}{l}\text { 'But we need to... you know, decide on what, what we think's important to change... and what's } \\
\text { changeable. And I don't think we can do anything about this [safety incident].' (GP, Practice A, APM) }\end{array}$ \\
\hline & & $\begin{array}{l}\text { Largely positive feedback } \\
\text { limited staff response }\end{array}$ & $\begin{array}{l}\text { 'We didn't have too many negatives [feedback] which is a good thing but also, it was sort of, well do we } \\
\text { need to change that much?' (PM, Practice A, Final interview) }\end{array}$ \\
\hline & & $\begin{array}{l}\text { Using staff identified areas } \\
\text { of service improvement } \\
\text { rather than patient } \\
\text { feedback }\end{array}$ & $\begin{array}{l}\text { '...even though it's not, it's not showing up as negative as I thought it might've, so I was really happy about } \\
\text { that, but I think the appointment system will still [need to be addressed]... And I think that will assist the, } \\
\text { there's less likely to be an error. So there's less likely to be a, ah, negative outcome for the patient' (PM, } \\
\text { Practice A, APM). }\end{array}$ \\
\hline \multirow[t]{9}{*}{$\begin{array}{l}\text { Barriers and } \\
\text { enablers to } \\
\text { intervention } \\
\text { development and } \\
\text { implementation }\end{array}$} & \multirow[t]{3}{*}{$\begin{array}{l}\text { Developing } \\
\text { interventions }\end{array}$} & $\begin{array}{l}\text { Intuitive problem-solving } \\
\text { process }\end{array}$ & $\begin{array}{l}\text { 'We're probably doing it anyway, but we don't realize it's a model for improvement.' (PN2, Practice C, Final } \\
\text { interview) } \\
\text { 'So we [other administration staff] we probably collaborate a lot. We throw ideas around. You know how to } \\
\text { do different things. So we're probably the thinkers.' (Admin, Practice F, Final interview) }\end{array}$ \\
\hline & & $\begin{array}{l}\text { Disconnect between staff } \\
\text { problem-solving process } \\
\text { and Mfl framework }\end{array}$ & $\begin{array}{l}\text { 'It was a good framework. Initially, what we found was when barriers kind of ah developed, we had trouble } \\
\text { readjusting to that [Mfl framework].' (GP, Practice F, Final interview) } \\
\text { 'I didn't ever use a model I was just sort of like, 'This is what I'm trying to achieve. This is how I'm going to } \\
\text { do it'... Did it work? Didn't it work? Which is probably the same model, but I just didn't actually outline it or } \\
\text { ever document it. It was just in my head.' (PN, Practice D, Final Interview) } \\
\text { 'We are not very keen of formally doing that [sic.] things [MFI]. The simple the better.' (GP, Practice E, Final } \\
\text { Interview) } \\
\text { '... we probably were never really good at documenting that stuff. Document ... as I said, in here you're kind } \\
\text { of doing things on the run, do you know what I mean? You go, 'Oh yeah, we'll do that.' (PM, Practice E, Final } \\
\text { Interview) }\end{array}$ \\
\hline & & $\begin{array}{l}\text { Integrating and adapting } \\
\text { problem-solving } \\
\text { approaches }\end{array}$ & $\begin{array}{l}\text { ' }[\text { The model for improvement) is a good process and it's simple but sometimes we complicate it by making it } \\
\text { bigger than what it is' (PN1, Practice C, Final Interview). } \\
\text { ' '... [we] do the PDSA cycle, not necessarily super formally but we just, we identify what needs to be done } \\
\text { and we try to make our changes small not big and then we introduce those to the practice or to specific } \\
\text { members of the practice team who might need to know about it.' (GP, Practice A, Final Interview) }\end{array}$ \\
\hline & \multirow[t]{6}{*}{$\begin{array}{l}\text { Implementing } \\
\text { interventions }\end{array}$} & Multidisciplinary team & $\begin{array}{l}\text { 'I just figured that it would end up falling probably on the three of us(PM, PN, Admin). Because I knew [GP] } \\
\text { was going to be time poor... So he was there if we needed him and we would bug him.' (PM, Practice B, } \\
\text { Final Interview) }\end{array}$ \\
\hline & & $\begin{array}{l}\text { Staff responsibility and } \\
\text { ownership for intervention } \\
\text { linked to type of } \\
\text { improvement activity }\end{array}$ & $\begin{array}{l}\text { 'I like data. I like playing with data [laughter]. I enjoyed doing a lot of the collection and stuff and seeing what } \\
\text { you can do to make it happen...' (PM, Practice C, Final Interview) }\end{array}$ \\
\hline & & $\begin{array}{l}\text { Difficulty in measuring } \\
\text { change in safety outcomes }\end{array}$ & $\begin{array}{l}\text { 'It is difficult to measure outcome because if you prevent a complication, it [is] what it is' (GP, Practice E, } \\
\text { Workshop 2) }\end{array}$ \\
\hline & & Use of soft measures & $\begin{array}{l}\text { '...because there were things that we couldn't really kind of quantify. I mean, how do you quantify [staff } \\
\text { member] stress level based on one particular aspect and you know separate it from...? That was what we } \\
\text { had trouble with, more than anything.' (GP, Practice F, Final Interview) }\end{array}$ \\
\hline & & $\begin{array}{l}\text { Staff support and } \\
\text { engagement }\end{array}$ & $\begin{array}{l}\text { 'Nobody wanted to be part of the safety improvement team, like, as soon as [you] mention anything like this, } \\
\text { everyone's just like [pause] 'Not again'.' (PM, Practice D, Workshop 2). }\end{array}$ \\
\hline & & Time and resources & $\begin{array}{l}\text { 'I felt as though we could have actually used a, 'Alright, what's going wrong? Let's troubleshoot this and } \\
\text { see.' I don't think as a team, we were able to devote the time or the resources or energy to actually do that } \\
\text { when we hit those barriers.' (GP, Practice F, Final Interview) } \\
\text { 'A lot's changed in the practice since we [started the trial]. A lot of fairly massive things. We've taken on } \\
50 \% \text { more students, we've got a few more extra learners, we've got a few other things going on plus we've } \\
\text { had just some stuff, health issues, which have had a huge impact.' (GP, Practice A, Final Interview) }\end{array}$ \\
\hline
\end{tabular}




\begin{tabular}{|c|c|c|c|}
\hline $\begin{array}{l}\text { Feasibility } \\
\text { measure }\end{array}$ & Theme & Subtheme & Participant quote \\
\hline \multirow[t]{2}{*}{ Trail scalability } & & $\begin{array}{l}\text { Increased facilitation and } \\
\text { support from research } \\
\text { team }\end{array}$ & $\begin{array}{l}\text { 'I think the workshops were valuable. I don't know whether we can just blame the [intervention barriers], } \\
\text { I suppose our lack of engagement with [the intervention]. Maybe if we had to engage a little bit more, it } \\
\text { probably would have kept us on track a bit more I think... even if it was just on the phone or something.' } \\
\text { (PM, Practice D, Final Interview) } \\
\text { 'I think you need somebody that's there as the overseer to keep us on track.' (PM, Practice B, Final } \\
\text { interview). }\end{array}$ \\
\hline & & $\begin{array}{l}\text { Real-time electronic } \\
\text { patient feedback } \\
\text { processes }\end{array}$ & $\begin{array}{l}\text { 'Something electronic I think we'd definitely be interested in. Even things, like the emails and text messages } \\
\text { and stuff to people after they've been to their appointment, people don't have to do them then and there. } \\
\text { They can sit on their couch at home and do it at night when they've actually got time... I would imagine we } \\
\text { would get different feedback if patients were being surveyed after their appointment.' (PM, Practice D, Final } \\
\text { interview). }\end{array}$ \\
\hline
\end{tabular}

$\mathrm{MFI}$, model for improvement.

\section{Acceptability}

\section{Intervention acceptability}

The majority of staff found the intervention acceptable. Staff reported that the intervention was predominantly positive and fitted within current organisational approaches to quality improvement.

\section{Attitude towards patient feedback on safety}

All staff valued patient feedback on safety. Positive feedback was welcomed and viewed as contributing to workplace morale, job satisfaction and reassurance that staff were meeting patient expectations. Feedback on safety was accepted when it aligned with staff awareness of issues. Furthermore, staff acceptance of the patient's reality also influenced believability of the feedback.

Staff exhibited a range of responses to negative patient feedback, including: acceptance, feelings of empathy, surprise or uncertainty, or being dismissive of feedback.

Commonly mentioned reasons for dismissing patient feedback involved unrealistic patient expectations; deeming patient concerns too problematic to fix or out of the practice's control; previous attempts to solve the problem have failed; or the patient was a known difficult patient (some staff speculated who a patient was even though the survey is anonymous).

\section{Using patient feedback to make changes}

Some staff were cautious about using the patient feedback for safety improvement activity. They contextualised the feedback in terms of where it may be coming from and how appropriate it would be to respond. Additionally, some mentioned difficulties in choosing priority areas to address due to largely positive patient scores limiting what they could respond to.

Four of the six practice teams saw this trial as a catalyst for undertaking improvements that aligned with previously identified staff priorities, and not responding directly to the patient feedback. Two practice teams attempted to link their chosen safety interventions back to domains of safety on the PC PMOS. For example, improving waiting time or availability of appointments was a focus area for staff yet the PC PMOS scores relating to access to care were largely positive. The other two practices did not attempt to link their previously identified target area to a
PC PMOS domain of safety. The remaining two practices chose to address areas that were directly related to areas of concern highlighted from the patient feedback. This was either a patient-reported safety incident or a negatively scored PC PMOS domain.

\section{Implementation of safety interventions Intervention fidelity}

The average intervention duration of 5.8 months was considered acceptable by most practice teams. Among the six practices, 25 safety improvement interventions were developed at the action planning meeting or during the implementation period. Of these, $17(68 \%)$ were fully implemented, $2(8 \%)$ partially implemented and $6(24 \%)$ not implemented.

The safety priorities targeted at the six practices included improvement in the following areas: communication of patient recall and reminders, access to equipment and supplies, access to care, accuracy of patient information, management of staff time, patient experience of waiting time, and patient knowledge of registrar skills and abilities. There were no differences observed in success of interventions that addressed either relational (communication, behavioural change, etc) or transactional issues (data cleaning, equipment and supplies, etc). Other mediating and contextual factors in the practice environment were attributed to the success or failure of safety interventions by staff.

\section{Barriers and enablers to intervention development and implementation \\ Developing interventions}

Staff employed both intuition and problem-solving processes to develop safety interventions. This process appeared to be an enabler for practice teams. This often took the form of a rapid and informal root cause analysis where common sense and a pragmatic approach was apparent. This process did not require external facilitation and staff were easily able to identify latent conditions in the practice that contributed to the safety concern. Staff reported regular use of this approach for safety and quality improvement activities unrelated to this project, but had not recognised it as formal improvement work. 
Some teams experienced challenges with translating their intuitive problem-solving approach onto the MfI framework. There was a perceived disconnect between the two problem-solving methods. This mainly related to adjusting to new habits or ways of working and adhering to a structured process. Practice teams with greater quality improvement experience were better able to integrate these approaches and adapt accordingly.

\section{Implementing interventions}

The high intervention fidelity shown in this trial was attributed to various factors. One key enabler was the multidisciplinary dynamic within the SIT. The teams largely consisted of a practice manager, administration staff member and a practice nurse. GPs adopted a more passive role in implementation. Nonetheless, GPs were engaged and supportive of the SIT and provided leadership and support when needed. Since most SITs comprised a practice manager, administration staff and a practice nurse, it was difficult to make comparisons about the effectiveness of teams that had different combination of staff roles.

Practice managers and administration staff often took primary responsibility and ownership for safety intervention implementation. As the interventions addressed the latent conditions within the primary care system that contribute to safety incidents, the corresponding activities and tasks often required input from administration staff rather than clinical staff. For example, ensuring patient demographic information was up to date or improving appointment scheduling were viewed as tasks to be undertaken by administration staff who are skilled and knowledgeable in this area.

Staff generally agreed that the MfI was a useful and familiar structure for implementing safety interventions. However, a few teams experienced some implementation challenges relating to the prescriptive nature and linear processes proposed in the model. Lack of model flexibility and adaptability were commonly cited as implementation barriers.

Staff also found measuring change difficult for various reasons. Identifying an appropriate measure directly relating to their safety intervention was challenging. For example, some staff indicated it was difficult to measure clinical outcomes or safety incidents averted. Often soft or proxy measures were used due to unavailability or inaccessibility of data.

Staff identified a number of other barriers to implementation. These were common across all practices and included lack of protected time, demanding priorities particularly for patient care, issues with staff recruitment and retention as well as staff leave, power and team dynamics, management support, and engagement from the wider practice.

\section{Scalability}

Staff recommended some improvements to the structure and components of the intervention that would enable
Box 1 Recommendations for intervention improvement

Simplification of intervention framework.

- Structured and defined intervention actions plans and corresponding safety measures for each of the primary care patient measure of safety domains of safety.

- Electronic data collection platforms to enable real-time patient feedback.

- Increased external intervention facilitation.

- Modification to questionnaire collecting patient-reported safety incidents.

future scale-up to a larger effectiveness trial or spread into policy and practice (box 1).

Existing practice infrastructure and resources were deemed adequate for participation.

The two learning workshops and facilitated action planning meeting with the research team were viewed as important. While the majority of staff felt that this level of facilitation was adequate, others suggested additional action planning meetings throughout the intervention phase would assist with accountability and implementation progress.

Patient data collection using the PC PMOS was considered relatively straightforward by practice staff. Only one practice (Practice A) failed to complete T2 data collection. Reasons for this included staff leave and patient survey fatigue. As the PC PMOS was a paper-based survey staff felt that improvements could centre on electronic data collection to increase the efficiency of real-time patient feedback, for example, via the use of waiting room iPads or emails to patients after their consultation.

\section{Secondary outcomes}

Patient feedback on contributing factors to safety: PC PMOS scores A total of $n=1750$ patients completed the PC PMOS at $\mathrm{T} 1$ and T2 (n=839 T1, n=911 T2), representing a practice mean of 140 and 182 at T1 and T2, respectively. The crude response rate was $10.7 \%$, however, the average response rate across the practices was $40.6 \%$. Patient characteristics are presented in table 2. Patients completing the PC PMOS were significantly more likely to be older and female (online supplementary appendix 2). Mean age was 56 years (SD 18.2) and mean number of visits to the practice in the previous 12 months was 8 (SD 8.6).

The PC PMOS total mean scores and domain scores for each practice at both times points are presented in table 3 . There was a significant increase in total mean PC PMOS score for all practices from $\mathrm{T} 1$ to $\mathrm{T} 2$ suggesting improved patient safety $(4.30 \quad(\mathrm{SD}=0.49) \quad$ to $4.37 \quad(\mathrm{SD}=0.47)$, $\mathrm{p}=0.002$ ). There were also significant increases in mean scores for all practices from $\mathrm{T} 1$ to $\mathrm{T} 2$ for the following domains: access to care (4.09 to 4.23, $\mathrm{p}<0.001$ ), communication (4.44 to $4.50, \mathrm{p}=0.018$ ), information flow (4.27 to $4.36, \mathrm{p}=0.007$ ) and patient-related factors (4.51 to 4.61, $\mathrm{p}<0.001)$. There was within and between practice variation for specific PC PMOS domain scores (table 3). 


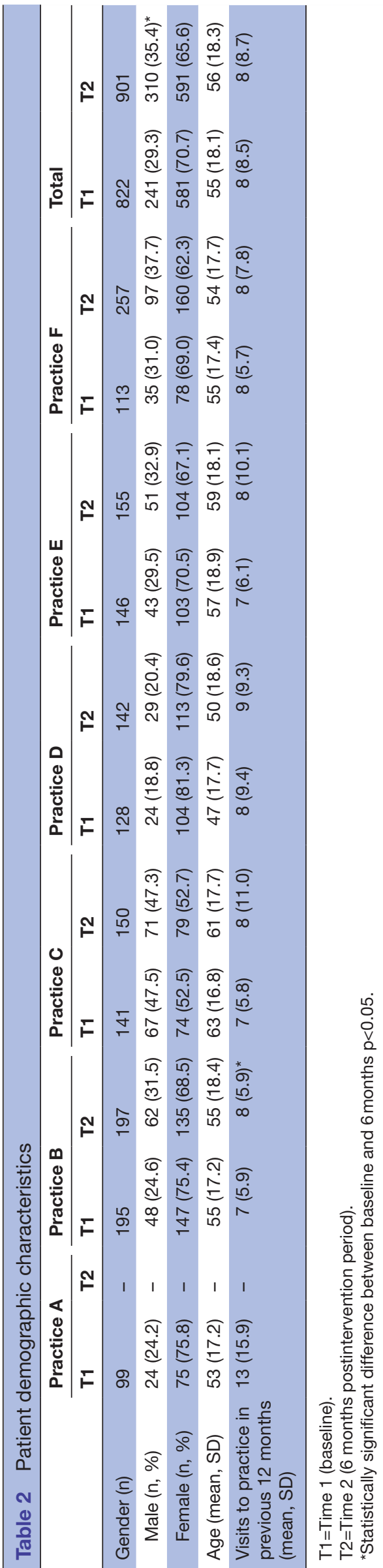

Patient-reported safety incidents and concerns data

Patient-reported safety incident data are presented in table 4 . There were $n=11$ patient-reported safety incidents at $\mathrm{T} 1$, and $\mathrm{n}=9$ at $\mathrm{T} 2$. The mean severity rating at $\mathrm{T} 1$ and T2 was 7.4 (scale 1-10 with 10 being 'extremely serious'). The median preventability rating of these safety incidents was 'definitely preventable' at T1, and 'probably preventable' at T2.

An additional $n=17$ safety incidents at $T 1$, and $n=12$ at $\mathrm{T} 2$ were identified from the 'other comments' section of the PC PMOS. Therefore, the total number of patientreported safety incidents was $n=28$ at $T 1$, and $n=21$ at T2. The number of patient-reported concerns (negative comments that were not a patient safety incident) decreased from $\mathrm{n}=45$ at $\mathrm{T} 1$ to $\mathrm{n}=25$ at T2 (table 4 ).

Practice measures of safety

Staff perceptions of safety culture

A total of $n=57$ staff completed the MOS survey at T1, and $\mathrm{n}=61$ at $\mathrm{T} 2$. For the total sample there was an increase in the mean percent positive score for the overall patient safety rating between $\mathrm{T} 1$ and $\mathrm{T} 2$, although not significant ( $72 \%$ to $74 \%, \mathrm{p}=0.851$ ). For the majority of the patient safety culture composites and the average across the ten composites there was a reduction in mean percent positive scores, with only one significant reduction for the Teamwork composite between $\mathrm{T} 1$ and T2 (89\%-80\%, $\mathrm{p}=0.029$ ) (online supplementary appendix 3 ).

\section{Safety incidents recorded on practice clinical risk management system}

There was a reduction in the number of incidents recorded on practice's clinical risk management system from T1 $(n=32)$ to T2 $(n=21)$ (online supplementary appendix 4). The incidents recorded on the practice clinical risk management system were different to the incidents reported by patients on the PC PMOS.

\section{DISCUSSION}

This is the first reported patient feedback on safety intervention in Australian primary care. The findings indicate that the intervention is feasible for scale-up to a larger effectiveness trial and further spread into policy and practice. Staff deemed the intervention acceptable, with minor recommendations for improvement. Intervention fidelity was high and implementation enablers were attributed to the intervention structure and framework, use of intuitive problem-solving approaches and multidisciplinary team involvement. Barriers to implementation reflected previously reported problems undertaking quality improvement in primary care, such as lack of time and staff, demanding priorities, power and team dynamics, and wider practice support and engagement. ${ }^{25-27}$ The process of systematically collecting patient safety data was achievable with $n=1750$ patient surveys completed. The utility of the PC PMOS tool as a measure for safety 


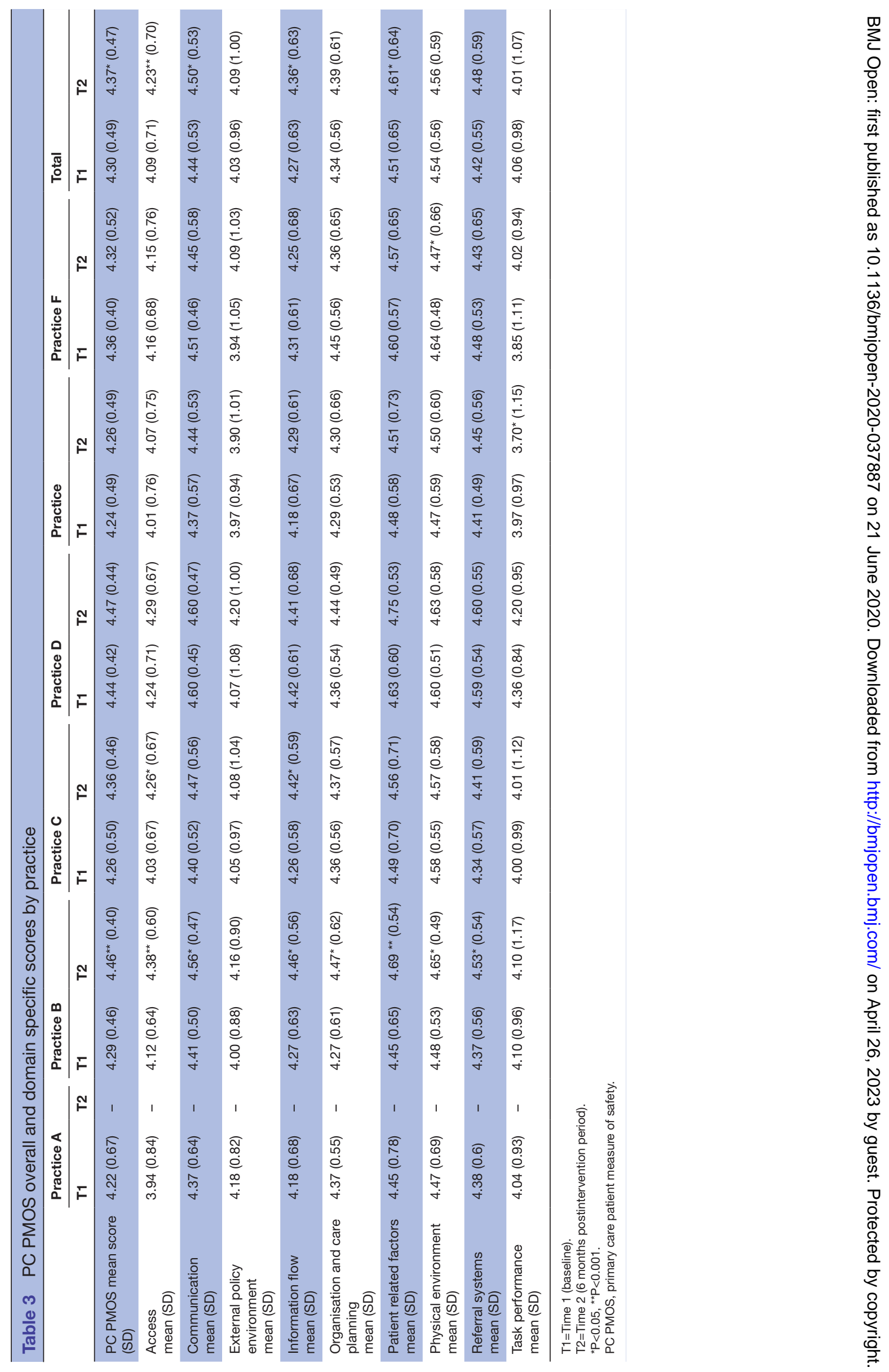




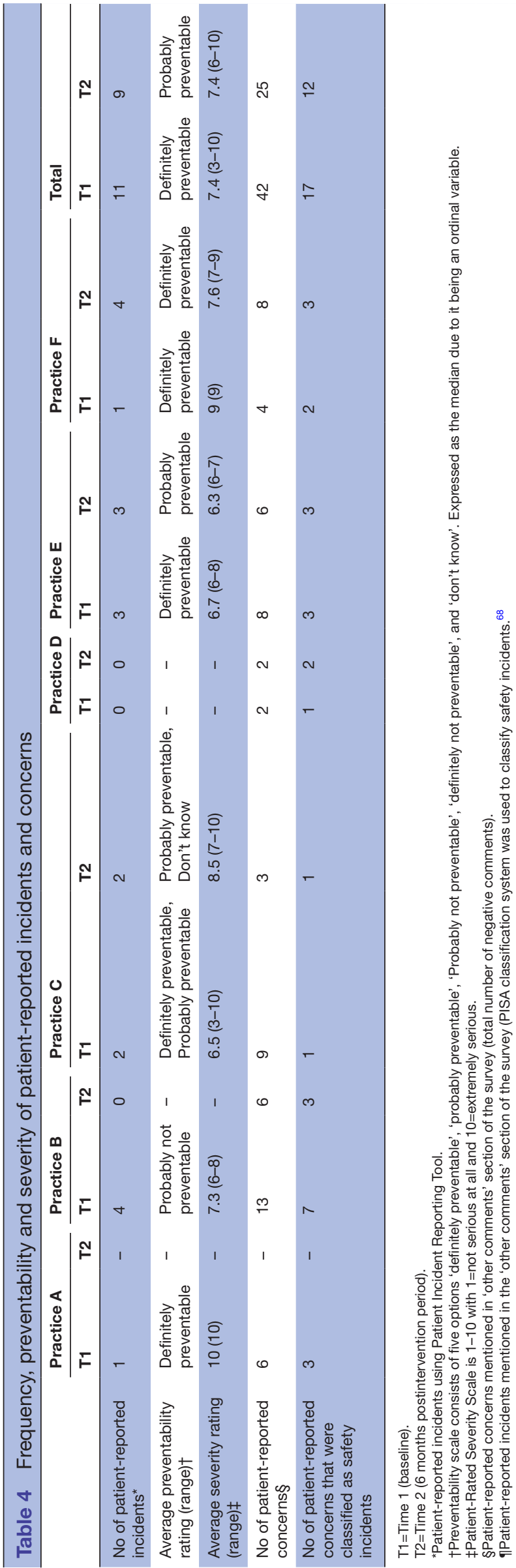

was demonstrated through the significant increase in mean scores for all practices from T1 to T2 (4.30 to 4.37, $\mathrm{p}=0.002$ ).

It is widely acknowledged that patient feedback is rarely used for safety and quality improvement purposes. ${ }^{38-46}$ This study identified some enablers and barriers that impacted on the intervention development and implementation including the team dynamic, improvement framework and staff attitude.

A unique aspect of this patient feedback on safety intervention was the multidisciplinary dynamic of the primary care teams, particularly administration staff leadership. This was considered a key enabler to intervention adherence and acceptability. The safety interventions targeted the contributing factors to safety incidents; as such, administration staff were ideally placed for intervention delivery. Administration staff transcended professional boundaries to generate engagement and support, and implement changes at the latent end of the primary care system. In this respect administration staff acted as change agents and innovators ${ }^{35} 4748$ and future safety improvement work should consider their current underused role.

Although the Mfi's PDSA cycle $^{31}$ is considered an effective, adaptable and flexible framework for quality improvement in some contexts, practice staff in this study identified it as a barrier to implementation. Formalising and documenting action plans in PDSA cycles was often in disconnect to their natural problem-solving approach and routine practice. In a time, resource and capacity scarce environment it is important that safety improvement frameworks are simple, and easily integrate or mimic everyday work flow. There are several well established quality improvement models ${ }^{4-51}$ that could be utilised for this patient feedback on safety intervention, however more research is needed to identify and investigate staff acceptability and appropriateness of the different frameworks in this context. ${ }^{52}$

Staff attitude towards patient feedback on safety was similar to previous research, which reveals staff difficulty to engage with or value patient feedback. ${ }^{36} 3841424553-57$ While staff described the value and benefit of seeking patient feedback on safety, this was not entirely reflected in action plans or translated during intervention implementation. More than half of the practice teams undertook safety interventions that were a priority for staff rather than a priority for the patient. Recommendations to improve staff action on patient feedback could centre on providing staff with structured and specific intervention examples that correspond to particular domains of safety on the PC PMOS. Moreover, such intervention examples could have explicitly linked measures of safety to each of the PC PMOS domains which may address the challenges staff experienced with creating measures of change. ${ }^{58}$

The process of systematically collecting primary care safety data from the practice, staff and patients was acceptable and feasible, yet some consideration is needed when determining appropriate measures of intervention 
effectiveness in a larger trial. Data about patient safety in primary care in Australia is largely absent. Australia does not have a structured or connected reporting and learning system to understand the threats to patient safety, and there is no current systematic way to collect information about safety incidents or patient harm. ${ }^{59} 60$ Using available sources of patient safety data in this study revealed some limitations; as such, objective measures of intervention effectiveness like statistical control charts, ${ }^{6162}$ PDSA cycle evaluation tools, ${ }^{6364}$ and record review ${ }^{6566}$ are recommended.

The findings from this study support results from other studies which have investigated patient feedback for safety improvement. While the majority of the research centres on hospital settings, the positive effect of patient feedback has been determined. ${ }^{38-12}$ One systematic review identified gaps in understanding regarding the enablers and barriers for implementation of patient feedback interventions. ${ }^{8}$ The findings from this study add to the discourse in this under-researched area.

A limitation of this study was the sample. The practices were from one regional area, which may limit the generalisability of the findings. However, the diversity within the practices was considered adequate for this feasibility study. All practices had participated in one or more of the Australian Primary Care Collaborative Programme $^{67}$ waves previously. Their commitment, interest and understanding of safety and quality improvement processes was potentially already elevated prior to study commencement when compared with other practices. Results suggest the merit of conducting a larger scale effectiveness-implementation trial to determine the translatability of this intervention programme and safety outcomes to primary care practices more generally.

\section{Conclusion}

This study's findings have demonstrated the feasibility of introducing an innovative patient feedback for safety improvement intervention in primary care, as well as contextual and intervention factors that promote safety improvement. The intervention complements existing safety improvement strategies and activities, and integrates into current patient feedback service requirements for primary care. Further research is needed to examine the intervention effects on safety incident reduction.

\section{Twitter Andrea L Hernan @andreahernan11}

Acknowledgements The authors acknowledge the practice staff and patients who participated in this study. The authors would also like to acknowledge the administrative support from Gillian Beard and Liz Jackway.

Contributors ALH conceived and designed the study, and was principal investigator for the study. SJG, KM, KK and VV contributed to the study design. ALH, SJG and HB were responsible for the qualitative data analysis and manuscript preparation. W was responsible for the quantitative data analysis and manuscript preparation. ALH created the first draft of the manuscript and was responsible for its revisions. SJG, HB, KM, KK, MJB and WV contributed to specific sections of the manuscript. All authors read and approved the final version of the manuscript.

Funding This work was supported by the Western Alliance Academic Health Science Centre (grant number WA-733721).
Competing interests None declared.

Patient and public involvement Patients and/or the public were involved in the design, or conduct, or reporting, or dissemination plans of this research. Refer to the Methods section for further details.

Patient consent for publication Not required.

Ethics approval Ethics approval was obtained from Deakin University Human Ethics Advisory Group, Faculty of Health. Project number: HEAG-H 175_2017.

Provenance and peer review Not commissioned; externally peer reviewed.

Data availability statement Data are available on reasonable request. Data are available on request to the authors.

Open access This is an open access article distributed in accordance with the Creative Commons Attribution Non Commercial (CC BY-NC 4.0) license, which permits others to distribute, remix, adapt, build upon this work non-commercially, and license their derivative works on different terms, provided the original work is properly cited, appropriate credit is given, any changes made indicated, and the use is non-commercial. See: http://creativecommons.org/licenses/by-nc/4.0/.

\section{ORCID iDs}

Andrea L Hernan http://orcid.org/0000-0003-4542-1186

Hannah Beks http://orcid.org/0000-0002-2851-6450

\section{REFERENCES}

1 Severinsson E, Holm AL. Patients' role in their own safety-a systematic review of patient involvement in safety. Open $\mathrm{J}$ Nurs 2015;05:642-53.

2 Sutton E, Eborall H, Martin G. Patient involvement in patient safety: current experiences, insights from the wider literature, promising opportunities? Public Management Review 2015;17:72-89.

3 Hall J, Peat M, Birks Y, et al. Effectiveness of interventions designed to promote patient involvement to enhance safety: a systematic review. Qual Saf Health Care 2010;19:e10-17.

4 World Health Organization. Patient engagement: technical series on safer primary care. Geneva: WHO, 2016.

5 Auraaen A, Slawomirski L, Klazinga N. The economics of patient safety in primary and ambulatory care: flying blind. OECD health working papers no 106. Directorate for employment, labour and social Affairs, health division: organisation for economic co-operation and development, 2018.

6 Health Foundation. Involving patients in improving safety. London, UK: The Health Foundation, 2013.

7 Vincent C, Davis R. Patients and families as safety experts. CMAJ 2012;184:15-16.

8 Park M, Giap T-T-T. Patient and family engagement as a potential approach for improving patient safety: a systematic review. J Adv Nurs 2020;76:62-80.

9 Berger Z, Flickinger TE, Pfoh E, et al. Promoting engagement by patients and families to reduce adverse events in acute care settings: a systematic review. BMJ Qual Saf 2014;23:548-55.

10 Birks Y, Hall J, McCaughan D, et al. Promoting patient involvement in safety initiatives. Nurs Manag 2011;18:16-20.

11 Lawton R, Armitage G. The role of the patient in clinical safety. London UK: Health Foundation, 2012.

12 Lawton R, O'Hara JK, Sheard L, et al. Can patient involvement improve patient safety? a cluster randomised control trial of the patient reporting and action for a safe environment (PRASE) intervention. BMJ Qual Saf 2017;26:622-31.

13 Sahlström M, Partanen P, Turunen H. Patient-Reported experiences of patient safety incidents need to be utilized more systematically in promoting safe care. Int J Qual Health Care 2018;30:778-85.

14 Stocks SJ, Donnelly A, Esmail A, et al. Frequency and nature of potentially harmful preventable problems in primary care from the patient's perspective with clinician review: a population-level survey in Great Britain. BMJ Open 2018;8:e020952.

15 Armitage G, Moore S, Reynolds C, et al. Patient-Reported safety incidents as a new source of patient safety data: an exploratory comparative study in an acute hospital in England. J Health Serv Res Policy 2018;23:36-43.

16 O'Hara JK, Reynolds C, Moore S, et al. What can patients tell us about the quality and safety of hospital care? findings from a UK multicentre survey study. BMJ Qual Saf 2018;27:673-82.

17 O'Hara JK, Isden R. Identifying risks and monitoring safety: the role of patients and citizens. Thought paper: The Health Foundation, 2013. 
18 Giles SJ, Lawton RJ, Din I, et al. Developing a patient measure of safety (PMOS). BMJ Qual Saf 2013;22:554-62.

19 Hernan AL, Giles SJ, Fuller J, et al. Patient and carer identified factors which contribute to safety incidents in primary care: a qualitative study. BMJ Qual Saf 2015;24:583-93.

20 Hernan AL, Giles SJ, O'Hara JK, et al. Developing a primary care patient measure of safety (PC PMOS): a modified Delphi process and face validity testing. BMJ Qual Saf 2016;25:273-80.

21 Hernan AL, Kloot K, Giles SJ, et al. Investigating the feasibility of a patient feedback tool to improve safety in Australian primary care: a study protocol. BMJ Open 2019;9:e027327.

22 Giles SJ, Parveen S, Hernan AL. Validation of the primary care patient measure of safety (PC PMOS) questionnaire. BMJ Qual Saf 2019;28:389-96.

23 Smith K, Baker K, Wesley D, et al. Guide to improving patient safety in primary care settings by engaging patients and families: environmental scan report. Rockville, MD: Agency for Healthcare Research and Quality, 2017.

24 Braithwaite J. Changing how we think about healthcare improvement. BMJ 2018;361:k2014.

25 Braithwaite J, Marks D, Taylor N. Harnessing implementation science to improve care quality and patient safety: a systematic review of targeted literature. Int J Qual Health Care 2014;26:321-9.

26 Brennan SE, Bosch M, Buchan $\mathrm{H}$, et al. Measuring organizational and individual factors thought to influence the success of quality improvement in primary care: a systematic review of instruments. Implement Sci 2012;7:121.

27 Kaplan HC, Provost LP, Froehle CM, et al. The model for understanding success in quality (MUSIQ): building a theory of context in healthcare quality improvement. BMJ Qual Saf 2012;21:13-20.

28 Nilsen P, Bernhardsson S. Context matters in implementation science: a scoping review of determinant frameworks that describe contextual determinants for implementation outcomes. BMC Health Serv Res 2019;19:189.

29 Greenhalgh T, Papoutsi C. Spreading and scaling up innovation and improvement. BMJ 2019;365:I2068.

30 Greenhalgh T, Papoutsi C. Studying complexity in health services research: desperately seeking an overdue paradigm shift. BMC Med 2018;16:95

31 Langley G, Moen R, Nolan K, et al. The improvement guide: a practical approach to enhancing organizational performance. John Wiley \& Sons, 2009.

32 Wright J, Lawton R, O'Hara J, et al. Chapter 6, learning from error: a comparative study of patient-reported patient safety incidents and existing sources of patient safety data. improving patient safety through the involvement of patients: development and evaluation of novel interventions to engage patients in preventing patient safety incidents and protecting them against unintended harm. Southampton (UK): NIHR Journals Library, 2016.

33 Agency for Healthcare Research and Quality. Medical office survey on patient safety culture. Rockville, MD, 2017.

34 Tavory I, Timmermans S. Abductive analysis: theorizing qualitative research. University of Chicago Press, 2014.

35 Sheard L, Marsh C, O'Hara J, et al. Exploring how ward staff engage with the implementation of a patient safety intervention: a UK-based qualitative process evaluation. BMJ Open 2017;7:e014558.

36 Sheard L, Marsh C, O'Hara J, et al. The Patient Feedback Response Framework - Understanding why UK hospital staff find it difficult to make improvements based on patient feedback: A qualitative study. Soc Sci Med 2017:178:19-27.

37 Carroll C, Patterson M, Wood S, et al. A conceptual framework for implementation fidelity. Implement Sci 2007;2:40.

38 Baldie DJ, Guthrie B, Entwistle V, et al. Exploring the impact and use of patients' feedback about their care experiences in general practice settings - a realist synthesis. Fam Pract 2017;35:13-21.

39 Sheard L, Peacock R, Marsh C, et al. What's the problem with patient experience feedback? A macro and micro understanding, based on findings from a three-site UK qualitative study. Health Expect 2019;22:46-53

40 de Vos MS, Hamming JF, Marang-van de Mheen PJ. The problem with using patient complaints for improvement. BMJ Qual Saf 2018;27:758-62.

41 Flott KM, Graham C, Darzi A, et al. Can we use patient-reported feedback to drive change? the challenges of using patient-reported feedback and how they might be addressed. BMJ Qual Saf 2017:26:502-7.

42 Boiko O, Campbell JL, Elmore N, et al. The role of patient experience surveys in quality assurance and improvement: a focus group study in English general practice. Health Expect 2015;18:1982-94.
43 Gardner K, Parkinson A, Banfield M, et al. Usability of patient experience surveys in Australian primary health care: a scoping review. Aust J Prim Health 2016;22:93-9.

44 Davies E, Cleary PD. Hearing the patient's voice? factors affecting the use of patient survey data in quality improvement. Qual Saf Health Care 2005;14:428-32.

45 Greenhalgh J, Dalkin S, Gooding K, et al. Functionality and feedback: a realist synthesis of the collation, interpretation and utilisation of patient-reported outcome measures data to improve patient care. Southampton (UK: NIHR Journals Library, 2017.

46 Gleeson H, Calderon A, Swami V, et al. Systematic review of approaches to using patient experience data for quality improvement in healthcare settings. BMJ Open 2016;6:e011907.

47 Greenhalgh T. Role of routines in collaborative work in healthcare organisations. BMJ 2008;337:a2448.

48 Lucas B, Nacer $\mathrm{H}$. The habits of an improver: thinking about learning for improvement in healthcare. London: The Health Foundation, 2015.

49 Toussaint JS, Berry LL. The promise of lean in health care. Mayo Clin Proc 2013;88:74-82.

50 Schroeder RG, Linderman K, Liedtke C, et al. Six sigma: definition and underlying theory*. J Oper Manage 2008;26:536-54.

51 Brannan KM. Total quality in health care. Hosp Mater Manage $Q$ 1998;19:1-8.

52 Johnson A, Clay-Williams R, Lane P. Framework for better care: reconciling approaches to patient safety and quality. Aust Health Rev 2019;43:653-5

53 Burt J, Campbell J, Abel G, et al. Programme grants for applied research. improving patient experience in primary care: a multimethod programme of research on the measurement and improvement of patient experience. Southampton (UK): NIHR Journals Library, 2017.

54 Wensing M, Vingerhoets E, Grol R. Feedback based on patient evaluations: a tool for quality improvement? Patient Educ Couns 2003;51:149-53.

55 Farrington C, Burt J, Boiko O, et al. Doctors' engagements with patient experience surveys in primary and secondary care: a qualitative study. Health Expect 2017;20:385-94.

56 Asprey A, Campbell JL, Newbould J, et al. Challenges to the credibility of patient feedback in primary healthcare settings: a qualitative study. Br J Gen Pract 2013;63:e200-8.

57 NIHR Dissemination Centre. Improving care by using patient feedback. In: Maxwell E, Lamont T, eds. NIHR themed review: National Institute for health research, 2019.

58 Woodcock T, Liberati EG, Dixon-Woods M. A mixed-methods study of challenges experienced by clinical teams in measuring improvement. BMJ Qual Saf 2019. doi:10.1136/bmjqs-2018-009048. [Epub ahead of print: 24 Aug 2019].

59 Russell LM, Dawda P. Patient safety in primary care: more data and more action needed. Med J Aust 2015;202:72-3.

60 Makeham M. Primary health networks - a new home for patient safety in primary care? Health Voices 2015;16:19-20.

61 Benneyan JC, Lloyd RC, Plsek PE. Statistical process control as a tool for research and healthcare improvement. Qual Saf Health Care 2003:12:458-64.

62 Fretheim A, Tomic O. Statistical process control and interrupted time series: a golden opportunity for impact evaluation in quality improvement. BMJ Qual Saf 2015;24:748-52.

63 Poots AJ, Reed JE, Woodcock T, et al. How to attribute causality in quality improvement: lessons from epidemiology. BMJ Qual Saf 2017;:26:933-7.

64 McNicholas C, Lennox L, Woodcock T, et al. Evolving quality improvement support strategies to improve Plan-Do-Study-Act cycle fidelity: a retrospective mixed-methods study. BMJ Qual Saf 2019;28:356-65.

65 Esmail A. Measuring and monitoring safety: a primary care perspective. thought paper. London: The Health Foundation, 2013.

66 Hanskamp-Sebregts M, Zegers M, Vincent C, et al. Measurement of patient safety: a systematic review of the reliability and validity of adverse event detection with record review. BMJ Open 2016;6:e011078.

67 Knight AW, Caesar C, Ford D, et al. Improving primary care in Australia through the Australian primary care Collaboratives program: a quality improvement report. BMJ Qual Saf 2012;21:94855.

68 Carson-Stevens A, Hibbert P, Williams $\mathrm{H}$, et al. Characterising the nature of primary care patient safety incident reports in the England and Wales national reporting and learning system: a mixed-methods agenda-setting study for general practice. Southampton (UK): NIHR Journals Library, 2016. 